\title{
Changes in the radiographic characteristics of the mandibular alveolar process in dentate women with varying bone mineral density: A 5-year prospective study
}

\author{
Grethe Jonasson $^{\mathrm{a}, *}$, Lisa Jonasson ${ }^{\mathrm{b}}$, Stavros Kiliaridis ${ }^{\mathrm{c}}$ \\ a Department of Orthodontics, Göteborg University, Box 450, SE 40530 Göteborg, Sweden \\ ${ }^{\mathrm{b}}$ École Polytechnique Fédéral de Lausanne, ELD 237, CH-1015 Lausanne, Switzerland \\ ${ }^{\mathrm{c}}$ Department of Orthodontics, Dental School, University of Geneva, Rue Barthélemy-Menn 19, CH-1211 Genève, Switzerland
}

Received 4 June 2005; revised 6 September 2005; accepted 7 October 2005

Available online 2 December 2005

\begin{abstract}
The association between skeletal bone mineral density (BMD) and mandibular alveolar bone mass has been reported to be rather weak, probably due to local functional factors. Many new investigations are therefore focused on assessing the mandibular bone structure. No long-term structural alterations have been reported in human mandibular bone with the exception of alveolar crest changes related to periodontal disease. The aim of this prospective study was to investigate dentate women to see if possible alterations in the radiographic characteristics of the mandibular alveolar bone are related to changes in BMD. The BMD of 131 women (initial age 22-75 years) was determined in the distal forearm with dual energy X-ray absorptiometry on two occasions separated by an interval of 5 years. Mandibular alveolar bone mass (MABM) was assessed both by the optical density and by the grey-level value on digitized, calibrated, periapical radiographs. The radiographic alveolar bone structure was evaluated with a visual index [Lindh C, Petersson A, Rohlin M. Assessment of the trabecular pattern before endosseous implant treatment: diagnostic outcome of periapical radiography in the mandible. Oral Surg Oral Med Oral Pathol Oral Radiol Endod 1996;82:335-43. [16]] and digitally by the alveolar bone texture. MABM decreased significantly during the 5-year period. Changes in MABM, evaluated by the mean greylevel value of a bone segment between the premolars, were correlated to changes in skeletal BMD $(r=0.33, P<0.001)$. Changes in MABM, evaluated by the optical density, did not correlate to changes in skeletal BMD. The overall trabecular pattern did not change during the study period, but small changes in the bone texture were measured. The changes in the bone texture were correlated with BMD change $(r=0.39$, $P<0.001)$. We conclude that changes in the mandibular alveolar bone do reflect changes in the skeletal BMD, and these may be estimated on periapical radiographs by changes in their grey-level value and their texture.
\end{abstract}

(C) 2005 Elsevier Inc. All rights reserved.

Keywords: Trabecular structure; Bone density; Humans; Mandible; Longitudinal studies; Texture analysis

\section{Introduction}

Conflicting results have been reported on the correlation between skeletal bone mineral density (BMD) and mandibular alveolar bone mass (MABM). In some studies, no association was found $[1,2]$, while others reported only a moderate one [3-6]. Furthermore, a number of cross-sectional studies have looked at the relation between skeletal BMD and mandibular

\footnotetext{
* Corresponding author. Viltstigen 12, SE-518 41 Sjömarken, Sweden. Fax: $+4633204337$.

E-mail address: grethe.jonasson@odontologi.gu.se (G. Jonasson).
}

bone structure [6-11], and four have reported a statistically significant correlation $[6,7,10,11]$. Longitudinal structural changes in the trabecular alveolar bone have been observed in ovariectomized rats after 1 year [12], but longitudinal human studies are needed to confirm if changes in BMD are reflected in oral bone loss and alterations in alveolar trabecular bone.

The aim of this prospective study was to investigate if possible alterations in the radiographic characteristics of the mandibular alveolar bone (bone mass, structure, alveolar thickness) in adult women are related to changes in skeletal BMD. 


\section{Material and method}

\section{Subjects}

One hundred and thirty-one dentate women, aged 22-75 years (mean $49.3 \pm 9.9 \mathrm{SD}$ ) at the initial recording, were consecutively asked to join the longitudinal study. All of them were attending a public dental service clinic, and none of them had any signs of current periodontal disease in the right mandibular section. Age distribution and anamnestic data affecting BMD are presented in Table 1. The mean number of teeth in the mandible at the initial recording was $13.6 \pm 1.6$ (range 9-16) and $13.5 \pm 1.8$ (range 8-16) at the final recording. Twelve women lost one mandibular tooth, and two women lost two mandibular teeth during the 5-year study period. The number of occluding pairs of teeth on the right lateral segment was $4.6 \pm 1.0$ pairs (1-6 pairs) on both the initial and the final occasion.

The sample described above originates from a total of 175 women who were consecutively asked to participate in the study, and they represent a sample of patients treated at their public dental clinic. One hundred and sixty women agreed to join the project. The initial inclusion criteria were no current periodontal disease and at least one mandibular premolar present. There was no history of recent dental trauma or orthodontic treatment that could have affected the bone and the roots. In the follow-up study, 24 women (out of 160) were not examined: 3 had died, 4 were seriously ill and unable to participate, 12 had moved out of the area and could not be reached, and 5 declined for other reasons. Furthermore, 5 women participated in both examinations but were excluded from the follow-up study because of either fracture of the nondominant arm (three individuals) or major changes in their dental status due to extensive prosthetic constructions (two subjects).

The Ethics Committee of Göteborg University, Sweden, approved the study. Informed consent was obtained from the participants.

\section{$B M D$ measurements}

BMD was determined in the distal forearm of the non-dominant arm by dual X-ray absorptiometry (Osteometer DTX-200, Osteometer, Rödovre, Denmark) Only women without any fractures in the non-dominant arm were included in the study. BMD was given as a percentage of the mean BMD of young women. Due to the error of the method, a cut-off value of $3 \%$ was used to define BMD change. A person with a bone loss of over $15 \%$ ( $3 \%$ per year) was defined as "a fast bone loser" [13].

\section{Alveolar bone mass}

A periapical radiograph (Kodak Ultra-Speed DF-58, Eastman Kodak, Rochester, NY, USA) of the premolar region was obtained by using stan-

Table 1

Age distribution and medical history affecting BMD in 131 women followed for 5 years

\begin{tabular}{lcc}
\hline Recording subjects & Initial recording $n$ & Five years after $n$ \\
\hline$<45$ years & $35(26.7 \%)$ & $19(14.5 \%)$ \\
$45-55$ years & $67(51.2 \%)$ & $45(34.4 \%)$ \\
$>55$ years & $29(22.1 \%)$ & $67(51.1 \%)$ \\
Premenopausal & $74(56.5 \%)$ & $40(30.5 \%)$ \\
Postmenopausal & $57(43.5 \%)$ & $91(69.5 \%)$ \\
HRT & $22(16.8 \%)$ & $31(23.7 \%)$ \\
Hysterectomy & $14(10.7 \%)$ & $19(14.5 \%)$ \\
Oophorectomy (unilateral) & $5(3.8 \%)$ & $5(3.8 \%)$ \\
Oophorectomy (bilateral) & $4(3.1 \%)$ & $7(5.3 \%)$ \\
Malabsorption syndromes & $9(6.9 \%)$ & $16(12.2 \%)$ \\
Treated for cancer & $6(4.6 \%)$ & $10(7.6 \%)$ \\
Hyperthyroidism & $5(3.8 \%)$ & $10(7.6 \%)$ \\
Active smokers & $29(22.1 \%)$ & $20(15.3 \%)$ \\
\hline
\end{tabular}

HRT-hormone replacement therapy, $n$-number of subjects, $\%$ - percentage of the subjects participating in the study.

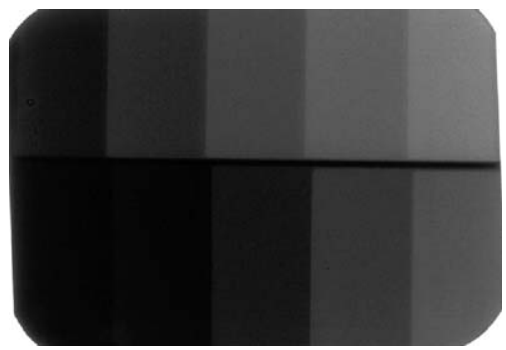

Fig. 1. A reference radiograph of the two separate step-wedges. The upper part is the image of the step-wedge with $10 \mathrm{~mm}, 8 \mathrm{~mm}, 6 \mathrm{~mm}, 4 \mathrm{~mm}$, and $3 \mathrm{~mm}$ steps. The lower part shows the $13 \mathrm{~mm}, 11 \mathrm{~mm}, 9 \mathrm{~mm}, 7 \mathrm{~mm}$, and $5 \mathrm{~mm}$ steps. The $6 \mathrm{~mm}$ step was used for calibration.

dardized paralleling technique. The dental X-ray apparatus was a long-cone Gendex Oralix 65S (Gendex Corp., Milwaukee, WI, USA). The exposure parameters were kept constant. The annual technical inspection showed no fluctuations in tube kilovoltage, and the variation in dose per exposure (reflecting tube current and exposure time) was under $2 \%$. Furthermore, test radiographs showed no heel effect from the X-ray apparatus. The exposure time was kept the same for all individuals. Directly after the patient exposure, a separate reference radiograph of two aluminum step-wedges was exposed in the absence of the patient using the same apparatus and the same exposure time. The step-wedges were placed directly on the film, which was placed on a 10-cm-thick acrylic plate in order to obtain a uniform background and to avoid background interference. The rationale for this procedure was to secure standardized conditions: the film was placed on a flat plate, and no other materials could exert any influence during exposure. In this way, the scattering of the X-ray beams would be as standardized as possible. The intraoral, the reference, and an unexposed radiograph were developed together in an automatic processing machine (Dent-X Model 410, Stamford, CT, USA). The unexposed radiograph used to control the quality of the processing procedure.

\section{Step-wedge}

Using a densitometer with an aperture of $\sim 2 \mathrm{~mm}$, it was possible to measure the optical density of a larger portion of bone than with a microdensitometer. However, this large aperture required large steps on the stepwedge. The radiographed step-wedge consisted of ten steps $(3-13 \mathrm{~mm}$ in thickness), which comprised the whole range in MABM (5.6-12.0 al. eq., $\mathrm{mm}$ ), and no extrapolation was necessary. The radiographic image of the step-wedge filled the whole area of the radiograph, and there were no problems to find space for the densitometer on any step (Fig. 1). There are several advantages in using separate step-wedges instead of a step-wedge attached to the film holder during patient exposure: (A) the image of the ten steps is large and distinct without any reflections from other structures. (B) There is no superposition of the step-wedge on the teeth, making it unnecessary to repeat the radiographic exposure of the patient. (C) The cheek has a heterogeneous tissue structure and a large variation in thickness both intraand interindividually. The varying thickness of the cheek does not influence the radiographic image of the step-wedge when the step-wedge is separated from the film holder and placed outside the mouth. In contrast, when the step-wedge is attached to the film holder, the cheek affects the different steps in various ways.

In the initial phase of the study, tests were performed in order to estimate the influence of the soft tissue of the cheek on the steps of a step-wedge included in a film holder. Large inter- and intraindividual variations were found in cheek thickness. The thinnest step placed most distally under the cheek was affected at least three times more than the step placed at the mouth angle. In order to standardize the radiographic image, we decided not to place the step-wedges in the mouth. Furthermore, it must be noted that it was very difficult to get good measurements for the steps in an "intraoral" step-wedge, due partly to anatomical structures and partly to artefacts such as shadows on the steps. Aware of the influence of the soft tissues, we consider that in a longitudinal study where the difference in optical density between two radiographs is calculated, the influence of the soft tissue is less important than in a cross-sectional study. 


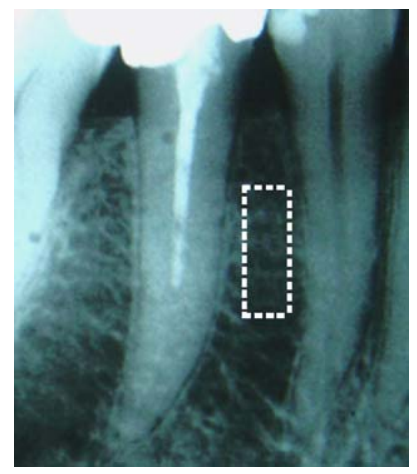

Fig. 2. The "rectangular tool " used to set the ROI when estimating the alveolar bone mass with the grey-level value.

Mandibular alveolar bone mass estimated by the optical density on analogue radiographs. The optical density of the exposed and processed radiographs was measured with a densitometer with an aperture of $\sim 2 \mathrm{~mm}$ (Macbeth TD500 , Macbeth Division of Kolmorgen Co., Newburgh, NY). The optical density of the alveolar bone was assessed as a mean of the optical density at six locations, three along each approximal side of the root of the first premolar. Care was taken to avoid the lamina dura and the apical area. If there was insufficient space between the teeth on the right side, the contra-lateral was used. If the first premolar was missing, the second premolar was used. Furthermore, the most crestal locations were situated at least $2 \frac{1}{2} \mathrm{~mm}$ from the crest to avoid any influence from bacterial plaque. The optical density of each step of the step-wedges was measured on the reference radiograph, and the values were plotted against the corresponding thickness of aluminum. The curve obtained provided the corresponding aluminum equivalents (al. eq., $\mathrm{mm}$ ) to the measured mean optical density of the alveolar bone. In this way, an indication of the mandibular alveolar bone mass (MABM) was obtained. The varying amount of soft tissue covering the hard tissue was not taken into consideration (see above). Due to the error of the method, a cut-off value of 0.40 al. eq., $\mathrm{mm}$ was chosen to define MABM change.

Mandibular alveolar bone mass estimated by the mean grey-level values of the alveolar bone on digitized radiographs. The radiographs were digitized with a resolution of 600 dpi by using Photo-Smart S20 (Hewlett Packard, Palo Alto, CA, USA). A pixel intensity value of 255 represented the lightest area on the film, and zero the black areas. The image density histogram in the Photoshop software (Adobe System, Edinburgh, Scotland) provided the mean grey-level value of the bone from each region of interest (ROI) on the radiographs of the subjects and of the $6 \mathrm{~mm}$ step of the step-wedge on the reference radiographs, which were used for calibration. In the Photoshop program, the ROIs were set on the apical radiograph of the individuals and on the $6 \mathrm{~mm}$ step of the reference radiograph with the "rectangular tool", avoiding the lamina dura and the most crestal locations (Fig. 2). No apical bone was included. The rectangular tool measured an area between the premolars situated halfway between the crestal and the apical area with the center $6 \mathrm{~mm}$ from the estimated cemento-enamel junction (Fig. 2). Replicated tests showed that there was a little variation in the mean grey-level value (less than $2 \%$ ) when the ROIs were set.

The site between the premolars is often used as a "standard site" for measuring MABM [14], since at the buccal side, no large muscle is inserted around the mental foramen. When the ROI was set with the rectangular tool between the premolars, the mylohyoid ridge was not included since it is situated more apically than in the molar area. The ROIs were not set in mandibles with very large tori, but if the torus was small (checked on dental casts), an ROI was set as near as possible to the standard site, avoiding torus. There is normally more space to set the ROIs between the first molar and the premolar than between the premolars, but at this site, the influence of the local functional factors is larger than at the premolar site [15]. The oblique and the mylohyoid ridges, being larger and more crestally situated in the molar area than in the premolar area also illustrate this. Due to the error of the method, a cut-off value of 4 was considered to be a change in grey-level values.

\section{Calibration}

Because of the differences in exposure and processing conditions, all radiographs were calibrated using the reference radiographs. We chose to adjust the brightness of the $6 \mathrm{~mm}$ thick step of the step-wedge to a mean grey-value of 60 (out of 256 grey-level values). After the reference radiographs were calibrated, the brightness of the radiographs of the alveolar bone was adjusted according to this calibration. Thus, if the $6 \mathrm{~mm}$ step had an initial grey-level value of 54, the brightness of both the reference radiograph and the radiograph of the individual was adjusted by +6 . If the $6 \mathrm{~mm}$ step was 68 initially, the brightness of both the reference radiograph and the radiograph of the individual was adjusted by -8 . In this way, we were able to compare changes during the 5-year period both intra- and interindividually.

\section{Structural changes}

Visual evaluation of the radiographic bone trabeculation. The trabecular bone pattern was assessed using a visual index proposed by Lindh et al. [16] and modified by Jonasson et al. [6]. Three periapical radiographs of the right premolar area of the mandible with varying trabeculation were used (Fig. 3) to assess the trabeculation pattern. With the help of these radiographs, the trabeculation of the alveolar process was classified as either sparse (regarded as an ordered numerical variable: grade 1), alternating dense and sparse (grade 2) or dense (grade 3). When the trabeculation was evaluated as sparse or dense, the whole radiographed area had the same degree of trabeculation. When the trabeculation was assessed as alternating dense and sparse, the trabecular network was not regular in coarseness: the trabeculation was normally denser cervically and sparser apically. Often, the trabecular network apically of the molars was extremely sparse or could be missing. Both the initial and the final radiographs were classified in this way.

Texture analysis of the trabecular bone pattern on digitized radiographs. The digitized calibrated radiographs were analyzed in Matlab (MathWorks, Natick, MA, USA) using traditional statistical description of the bone texture in the image in a specially developed program. Texture is defined as a global pattern arising from the repetition, either deterministically or randomly, of local subpatterns. A radiograph represents a two-dimensional projection of the trabecular bone architecture, and the pattern of these projections is the texture in the image. The "local subpatterns" in our program are features such as the repeated occurrence of certain grey-scale configurations (transition from trabeculae to intertrabecular spaces, edges, and spots), extracted from 36 radiographs with "typical bone trabeculation". These features were then used to classify the images into four groups. Features from 10 radiographs of sparse trabeculation (grade 1 in the visual evaluation) were extracted to characterize group A. Features from 10 radiographs with alternating dense and sparse trabeculation having thin trabeculae (grade 2 in the visual evaluation) were
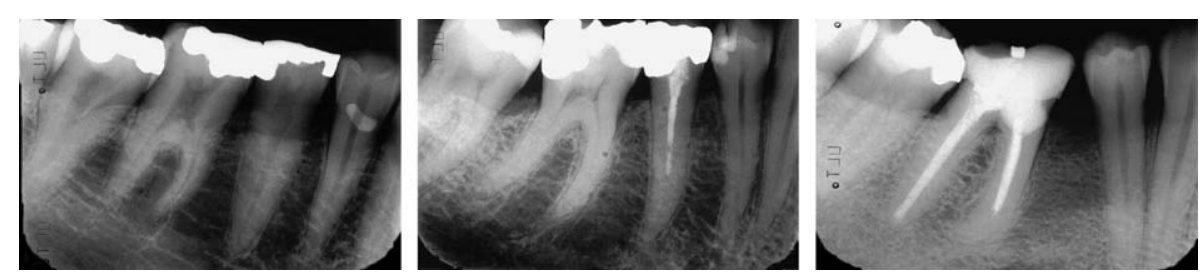

Fig. 3. Reference images presenting the trabecular pattern as sparse trabeculation, alternating dense and sparse trabeculation, and dense trabeculation. 


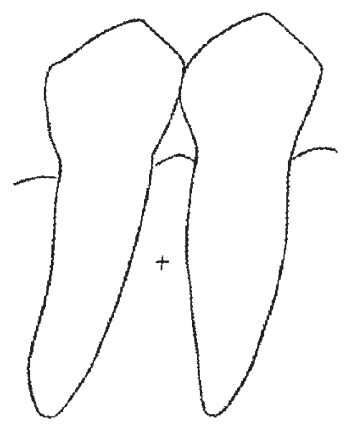

Fig. 4. The site for measuring the interdental thickness between the premolars, 6 $\mathrm{mm}$ from the alveolar bone crest.

extracted to characterize group B. Features from 6 radiographs with alternating dense and sparse trabeculation having thick trabeculae (grade 2 in the visua evaluation) characterized group C. Features from 10 radiographs with dense trabeculation (grade 3 in the visual evaluation) characterized group D. These selected radiographs form a training set for the program and are used to set weights in the classification algorithm. With these weights known, any new image can be classified as one of the four subgroups with a simple linear classifier by extracting the same features as in the training set. The analyzed bone was selected with a rectangular tool in the standard area, between the premolars, halfway between the crest and the apical area. All radiographs were calibrated to compensate for differences in exposure and development procedures as mentioned above, but otherwise, their grey-level values were not changed. In this way, the attenuation of the X-ray beams due to the buccal and lingual cortices was "preserved".

\section{Changes in mandibular bucco-lingual dimension}

The bucco-lingual thickness of the mandibular alveolar process was measured on dental casts with a dial calliper (Kori-HSL 6871-0201 range 0-15 mm or Kori-HSL 250-00 range 0-10 mm, Kori Seiki MFG. Co. Ltd., Tokyo, Japan) on a location between the mandibular premolars and between the molar and the premolar, $6 \mathrm{~mm}$ from the estimated cemento-enamel junction, according to Jonasson et al. [6,17] (Fig. 4). The alveolar thickness was taken into consideration because of its possible influence on the bone mass [6]. The alveolar thickness was a separate variable in the statistical analyses. It was not used correctively (MABM/alveolar thickness) since this variable did not perform better than MABM alone.

\section{Statistical methods}

A paired $t$ test was used to detect the 5-year changes in body weight, BMI, BMD, MABM, and grey-level values. The Chi-squared test was used to detect if there was any significant difference over time regarding the bone texture. The Pearson correlation coefficient $(r)$ was used to determine the amount of association between changes in MABM, mean grey-level value of the bone, alveolar thickness, and BMD. Spearman's rank correlation coefficient $\left(r_{\mathrm{s}}\right)$ was used when the trabeculation and the bone texture were involved. Stepwise multiple regression analyses were used to take into account possible covariates, such as body weight, BMI, alveolar thickness, and age.

Table 2

The random error calculated according to Dahlberg's formula and the reproducibility calculated according to a method proposed by Houston [18] after duplicate measurements in 20 cases

\begin{tabular}{lll}
\hline & Random error & Reproducibility (\%) \\
\hline BMD (\%) & 1.17 & 98.1 \\
MABM (al. eq., mm) & 0.15 & 98.0 \\
Grey level (1-256 steps) & 1.04 & 98.7 \\
Interdental thickness (mm) & 0.09 & 97.8 \\
\hline
\end{tabular}

\section{The error of the method}

The random error of the method was calculated according to Dahl berg's formula: $S_{e}=\sqrt{\frac{\sum d^{2}}{2 n}}$, where $d$ is the difference between two measurements in 20 cases. The reproducibility was calculated according to a method proposed by Houston [18]: $1-S_{\mathrm{e}}^{2} / S_{\mathrm{i}}^{2}\left(S^{2}{ }_{\mathrm{i}}\right.$ is the total variance of the measurement). The random error and the reproducibility of the measurements are shown in Table 2. The trabeculation was assessed twice in 24 subjects by one observer (GJ) with an interval of a week, and the strength of agreement was very good $($ kappa $=0.89)$. Paired $t$ tests between the first and the second evaluations of all methods tested revealed no systematic error between the two occasions.

\section{Results}

During the 5-year period, the mean body weight and the mean BMI of the sample increased and mean BMD decreased $(P<0.001$, Table 3$)$. However, during the 5 -year period $12.5 \%$ of the participants increased their BMD more than $3 \%$ and $36.2 \%$ lost more than $3 \%$. Of these, three women $(2.3 \%)$ lost more than $15 \%$ in BMD.

\section{Alveolar bone mass}

Mandibular alveolar bone mass estimated by optical density of analogue radiographs

Mean MABM decreased during the 5-year period $(P<0.001$, Table 3$)$. In $22.0 \%$ of the subjects, it increased, and in $44.2 \%$, it decreased more than $0.40 \mathrm{~mm}$ al. eq.

Mandibular bone mass estimated by the mean grey-level value of the alveolar bone on digitized radiographs

The mean grey-level value decreased during the 5-year period $(P<0.05$, Table 3$)$. In $31.9 \%$ of the subjects, it

Table 3

Initial and final values and 5-year changes in general individual characteristics and mandibular alveolar bone measures in 131 women

\begin{tabular}{|c|c|c|c|c|c|c|c|c|}
\hline & \multicolumn{2}{|l|}{ Initial } & \multicolumn{2}{|l|}{ Final } & \multirow[t]{2}{*}{$P$} & \multicolumn{2}{|c|}{$\begin{array}{l}5 \text {-year } \\
\text { Change }\end{array}$} & \multirow[t]{2}{*}{ Range } \\
\hline & Mean & SD & Mean & SD & & Mean & SD & \\
\hline \multicolumn{9}{|l|}{ General data } \\
\hline Weight (kg) & 65.0 & 9.59 & 66.8 & 9.73 & $<0.001$ & 1.79 & 4.210 & $\begin{array}{l}-12 \\
\text { to } 18\end{array}$ \\
\hline BMI & 23.7 & 3.42 & 24.3 & 3.34 & $<0.001$ & 0.36 & 1.792 & -6 to \\
\hline BMD (\%) & 86.9 & 12.63 & 84.7 & 13.91 & $<0.001$ & -2.30 & 5.217 & $\begin{array}{l}-22 \\
\text { to } 11\end{array}$ \\
\hline \multicolumn{9}{|l|}{ Alveolar bone } \\
\hline $\begin{array}{l}\text { MABM } \\
\quad \text { (al. eq., mm) }\end{array}$ & 8.2 & 1.26 & 7.8 & 1.30 & $<0.001$ & -0.44 & 1.037 & $\begin{array}{l}-2.3 \\
\text { to } 2.9\end{array}$ \\
\hline $\begin{array}{l}\text { Grey level } \\
(0-255)\end{array}$ & 74.0 & 15.93 & 71.6 & 18.04 & $<0.05$ & -2.40 & 12.722 & $\begin{array}{l}-23 \\
\text { to } 34\end{array}$ \\
\hline $\begin{array}{l}\text { Int.thick. } \\
\quad 6-5(\mathrm{~mm})\end{array}$ & 10.4 & 1.13 & 10.0 & 0.96 & $<0.001$ & -0.32 & 0.384 & $\begin{array}{l}-1.4 \\
\text { to } 0.3\end{array}$ \\
\hline $\begin{array}{l}\text { Int.thick. } \\
\text { 5-4 (mm) }\end{array}$ & 8.8 & 1.12 & 8.6 & 1.11 & $<0.001$ & -0.23 & 0.365 & $\begin{array}{l}-1.4 \\
\text { to } 0.9\end{array}$ \\
\hline
\end{tabular}

MABM is estimated using six sites. Grey-level value is measured between the premolars. "Int.thick. 6-5" denotes the interdental bucco-lingual thickness between the first molar and the second premolar and "Int.thick. 5-4" between the premolars, $P$ is calculated by using paired $t$ test. 
Table 4

Correlations between BMD change and change in MABM, grey-level value, texture, CEJ-AC, and alveolar thickness in 131 women

\begin{tabular}{llll}
\hline & & $r$ & $P$ \\
\hline$\Delta$ BMD & $\Delta$ MABM & 0.10 & ns \\
$\Delta$ BMD & $\Delta$ Grey level & 0.33 & $<0.001$ \\
$\Delta$ BMD & $\Delta$ Texture & $0.39^{\mathrm{a}}$ & $<0.001$ \\
$\Delta$ BMD & $\Delta$ Int.thick 6-5 & 0.29 & $<0.01$ \\
$\Delta$ BMD & $\Delta$ Int.thick 5-4 & 0.10 & ns \\
\hline
\end{tabular}

ns: no significance $(P>0.05)$.

MABM is estimated at six sites. Grey-level value is measured between the premolars. "Int.thick. 6-5" denotes the interdental bucco-lingual thickness between the first molar and the second premolar and "Int.thick. 5-4" between the premolars, $r$ is Pearson's correlation coefficient.

a Spearman's rank correlation coefficient.

increased, and in $35.1 \%$, it decreased more than 4 steps (out of 256 grey-level values). The change in grey-level value, measured between the premolars, was correlated to the change in BMD ( $r=0.33, P<0.001$, Table 4$)$, to the MABM change ( $r=0.36, P<0.001$, Table 5) but not to the change in interdental alveolar thickness.

\section{Structural changes}

Visual evaluation of the radiographic bone trabeculation

In all but three women, the overall trabecular pattern did not change during the 5-year period (Fig. 5). Two of them were fast bone losers and lost more than 0.40 al. eq. in MABM (0.70 and $0.41)$.

Texture analysis of the radiographic trabecular pattern on digitized radiographs

The distribution of the bone texture at the initial and the final recording was characterized by a higher frequency of group A and $\mathrm{B}(>75 \%$ of the participants), corresponding to sparse trabeculation and alternating dense and sparse trabeculation with thin trabeculae (Fig. 6). The changes in bone texture over the 5-year period were not statistically significant. Certain individuals "increased" in bone texture (changed to a group characterized by a denser trabeculation than before), some "decreased". The change in texture was significantly correlated to the BMD change $\left(r_{\mathrm{s}}=0.39, P<0.001\right.$, Table 4$)$, to the

Table 5

Correlations between MABM change and change in grey-level (rectangular tool), texture, CEJ-AC, and alveolar thickness in 131 women

\begin{tabular}{llll}
\hline & & $r$ & $P$ \\
\hline$\Delta$ MABM & $\Delta$ Grey level & 0.36 & $<0.001$ \\
$\Delta$ MABM & $\Delta$ Texture & $0.28^{\text {a }}$ & $<0.01$ \\
$\Delta$ MABM & $\Delta$ Int.thick. 6-5 & 0.14 & ns \\
$\Delta$ MABM & $\Delta$ Ínt.thick. 5-4 & 0.10 & ns \\
\hline
\end{tabular}

ns-no significance $(P>0.05)$.

"Int.thick 6-5" denotes the interdental bucco-lingual alveolar thickness between the first molar and the second premolar and "Int.thick 5-4" between the premolars, $r$ is Pearson's correlation coefficient.

a Spearman's rank correlation coefficient.

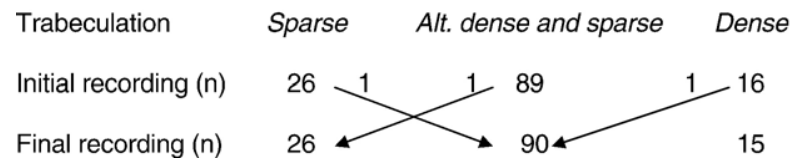

Fig. 5. Number of women with sparse, alternating dense and sparse, plus dense trabeculation at the first and second recording in 131 women.

change in MABM $\left(r_{\mathrm{s}}=0.28, P<0.01\right.$, Table 5), and change in mean grey-level value $\left(r_{\mathrm{s}}=0.24, P<0.05\right)$. The change in bone texture was not correlated to the change in interdental alveolar thickness.

Changes in bucco-lingual dimension of the mandibular alveolar bone. The mean interdental thickness, between the premolars and between the first molar and the second premolar, decreased significantly during the 5 -year period $(P<0.001$, Table 3). There was a significant correlation between the BMD change and the decrease in the interdental alveolar thickness between the first molar and the second premolar $(r=0.29$, $P<0.01$ ) but not between the premolars (Table 4).

\section{Discussion}

In this 5-year prospective study, we found that mean BMD decreased significantly, and that alterations in the mandibular alveolar bone (bone mass, structure, bucco-lingual alveolar thickness) were related to the BMD changes. The factors that may have influenced BMD during the study period (change of diet, physical activity, hormone replacement therapy, menopause) were not considered, as this was not within the scope of the investigation. Our study was limited to a 5-year followup period, and the variation in the subjects' health and age was large, but the majority were perimenopausal women. At this age, the deficiency in estrogen induces an increased bone turnover $[19,20]$ with an increased number of remodeling sites, increased erosion depth and impaired bone formation in each remodeling circle, leading to a net skeletal bone loss.

The golden standard for fracture risk is BMD of the proximal femur measured with dual X-ray absorptiometry [21]. The present study is not large enough to assess fracture risk, and its focus was to compare skeletal bone loss with mandibular changes. Both the forearm and the mandible are "cortical"

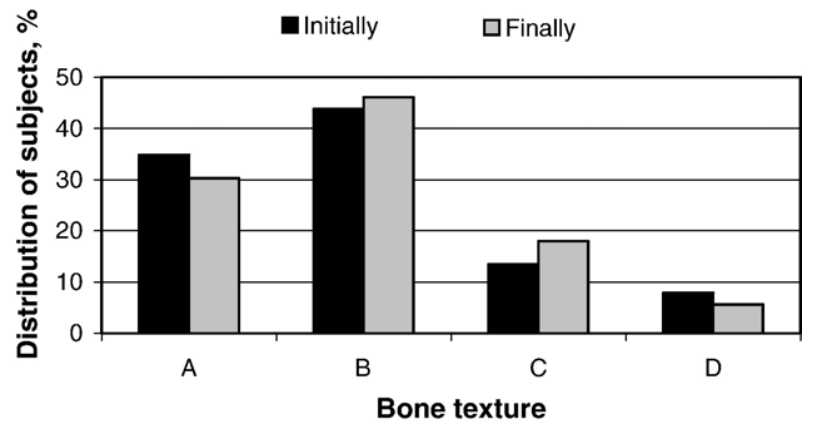

Fig. 6. Initial and final bone texture in percentage in the four groups (A, B, C, D). Group A: sparse trabeculation, Group B: alternating dense and sparse trabeculation and thin trabeculae, Group C: alternating dense and sparse trabeculation and thick trabeculae, Group D: dense trabeculation. 
bones, with approximately $80 \%$ cortical and $20 \%$ trabecular bone $[22,23]$, and it is therefore reasonable to compare them. Furthermore, longitudinal studies have shown that the most pronounced bone loss after the menopause occurs in the forearm $[24,25]$.

\section{Alveolar bone mass}

The measures estimating mandibular bone mass, that is MABM and the grey-level value, decreased significantly during the study. The changes in BMD were associated with changes in the grey-level value of the mandibular alveolar bone measured between the premolars, but not with changes in MABM. Several factors may explain this discordance. First of all, it could be due to the variation in size and place of the selected sites. MABM was measured at 6 sites around the first premolar in circles with $2 \mathrm{~mm}$ in diameter. The rectangular tool, used when the grey-level value was assessed, included a larger area than the circle. It was placed halfway between the crest and the apical area, which is the area shifting from a denser cervical trabeculation to a sparser trabeculation in the group with alternating dense and sparse trabeculation. Furthermore, the grey-level values were all assessed in the same period with the two radiographs placed side by side, whereas the optical density was assessed in two periods 5 years apart. The method for estimating MABM by its optical density is obsolete, but assessing the mean grey-level value at the standard site calibrated with a separate step-wedge is more promising for the future.

Whereas the deficit in bone formation in cortical bone results in an increased number of lacunae and porosities and later on in thinner cortical plates $[4,10,11]$, it leads to larger intertrabecular spaces and a thinning of the trabeculae in the cancellous bone [26]. When the cortices and trabeculae are thinner and the intertrabecular spaces are larger in a certain area (ROI), the mineral content is decreased, and therefore, also the grey-level value of the radiographed area is decreased.

\section{Structural changes}

Only in three individuals did the overall trabecular pattern, evaluated by the visual index, change. Two of those individuals were fast bone losers. If the study period had been longer, it is highly possible that we would have seen more pairs of radiographs where the trabecular pattern had changed. In contrast, the category of the bone texture changed during the study period in several cases. The observed changes in bone texture possible reflect small changes in size of the intertrabecular spaces, which are difficult to observe visually on the radiographs. The computer program for evaluating the bone texture may be improved in the future by training it with autopsy bone segments of known bone density from dual energy X-ray absorptiometry (golden standard) and when turned from an ordinal to a continuous scale. The local functional factors will always complicate the use of the mandible to evaluate skeletal bones, but a rough visual index (as Lindh's index) can be used to screen patients with sparse trabeculation although the method is not sensitive enough to record longitudinal changes.

Radiographs are a two-dimensional projection of the threedimensional trabecular network. It is therefore debated both how well the radiographic image represents the "true" trabecular network and from which part of the trabecular bone the image is created. Based on studies of experimental focal bone loss in radius, the radiographically apparent trabecular structure has been attributed mainly to the junction area between the cortical bone and the trabecular bone [27]. A bone defect in that region is visible in radial radiographs, whereas defects in the "interior" trabecular bone are not detectable. However, in a recent study in cadaver specimens, the trabecular bone in the alveolar process was found to account for most of the visible fine structures in the dental radiographs [28]. The interdental alveolar bone is thinner than the radius, so it is possible that it is less important where the radiographic trabecular pattern is created in our selected area between the premolars than in the radius and at the root apices [29].

Direct measurements of trabecular width and separation/ intertrabecular space have shown that the increase in distance between adjacent trabeculae accounted for more than double the age-related bone loss compared to the decrease in trabecular width [26]. Patients with osteoporosis had a reduction in the area and the periphery of the trabeculae and a reduction in the complexity of the trabecular pattern in the posterior alveolar bone in comparison with controls [7]. Similarly, a significant difference in skeletal BMD has been reported between individuals with dense, alternating dense and sparse, and sparse trabeculation [6]. Thus, it seems that especially, the intertrabecular spaces increase in osteopenic women, resulting in fewer edges and transitions (from trabeculae to intertrabecular spaces) in the ROIs leading to a change in bone texture.

\section{Changes in bucco-lingual alveolar bone dimension}

The change in the bucco-lingual thickness of the alveolar process was significantly correlated to change in BMD but not to the change in alveolar bone mass. The changes measured in the alveolar thickness may reflect changes in the alveolar bone provided that there is no age-related difference in the thickness of the gingival and the periodontal ligament. Probably there is a threshold to be passed before the negative systemic factors (increasing age, deficit in hormones, osteoporosis) can change the mandibular bone dimension due to the local functional forces: the masseter muscle and the number of occluding teeth. These local factors may stimulate the bone and counteract local bone loss, at least as long as the masseter muscle keeps its functional strength [30,31], and the maxillary antagonist teeth are preserved $[32,33]$. In this way, the mandibular bone alterations due to the systemic factors might be counteracted [15]. Another explanation for the lack of correlation between changes in mandibular bone mass and changes in alveolar thickness may be that there is a time delay in dentate areas between the two events [34]. In contrast, rapid changes $(8$ weeks) in the bucco-lingual dimension of the alveolar bone have been measured in extraction sockets in an experimental 
study in dogs [35] and after 4 months in a clinical study of immediate implant placement in humans [36]. It was found that the buccal bone dimension had undergone a "horizontal" resorption that amounted to about 56\%, whereas the corresponding reduction of the lingual cortex was 30\% [36]. This rapid decrease in the bucco-lingual dimension of the alveolar mandibular process directly after tooth extraction $[35,36]$ demonstrates a periosteal resorption specific to the alveolar process, and to our knowledge, it has not been reported regarding any other bone. It is supported by findings in anatomical specimens where remodeling fields of "resorptive nature" have been found on the alveolar edentulous crest of adult mandibles [37]. The most reasonable explanation for the measured mean decrease in the bucco-lingual dimension of the alveolar process is a slow periosteal resorption in the dentate region.

\section{Implications}

In rat studies, it has been found that $50-75 \%$ of the strength of the bone is determined by its external geometry, its bone mineral density (BMD) and the trabecular bone structure [38]. It is well known that the alveolar bone decreases in size after tooth extraction, and in the present study, we saw that the mandibular alveolar bone decreased in size in dentate areas too. We saw an age-related decrease in alveolar bone mass that is in contrast to what has been observed in the mandibular basal bone, which increases in density with aging [39].

The participants answered a questionnaire about their medical history and life style factors. No single parameter from this questionnaire was correlated to BMD and BMD change, but it is important to consider the existence of several known risk factors such as previous fractures, weight loss, certain medication and decreased physical activity together with the radiographic findings and other clinical observations such as the alveolar thickness and shape [17] if an effective probability estimate of future osteoporotic fractures is to be made on the basis of oral findings [40]. Osteoporotic fractures are not a problem in the jaws, but an evaluation of the alveolar bone structure with Lindh's index together with an appropriate questionnaire may be a valuable help in many situations. A periapical radiograph showing dense trabeculation indicates the need of increased prudence when drilling for implants due to increased heating and consequently an increased risk of local necrosis [41]. A periapical radiograph with sparse trabeculation indicates a warning in implant treatment and a need of cortical fixation and a prolonged healing process before the implant can be loaded [42]. Furthermore, in adult orthodontics, a different tissue reaction could be expected when moving teeth in bone with sparse or dense trabeculation $[43,44]$.

\section{Conclusion}

Changes in the mandibular alveolar bone reflect alterations in the skeletal BMD though the correlations are weak, and they can be observed on periapical radiographs by changes in their grey-level value and in the bone texture. Age-related changes were detected in the alveolar bone in the horizontal dimensions after 5 years. The changes in the bucco-lingual dimension are correlated to changes in BMD. The overall trabecular pattern does not change in a 5-year period in perimenopausal women, but a small deterioration occurs in bone texture.

\section{Acknowledgments}

This study was made possible by a grant from the R\&D center in Southern Alvsborg County, Sweden.

\section{References}

[1] Mohajery M, Brooks SL. Oral radiographs in the detection of early signs of osteoporosis. Oral Surg Oral Med Oral Pathol 1992;73:112-7.

[2] Southard KA, Southard TE, Schlechte JA, Meis PA. The relationship between the density of the alveolar process and that of post-cranial bone. J Dent Res 2000;79:964-9.

[3] Von Wowern N, Stoltze K. Histoquantitation on small jaw specimens. Scand J Dent Res 1978;86:193-9.

[4] Kribbs PJ, Chesnut III CH, Ott SM, Kilcoyne RF. Relationships between mandibular and skeletal bone in an osteoporotic population. J Prosthet Dent 1989;62:703-7.

[5] Jacobs R, Ghyselen J, Koninckx P, Van Steenberghe D. Long-term bone mass evaluation of mandible and lumbar spine in a group of women receiving hormone replacement therapy. Eur J Oral Sci 1996;104:10-6.

[6] Jonasson G, Bankvall G, Kiliaridis S. Estimation of skeletal bone mineral density by means of the trabecular pattern of the alveolar bone, its interdental thickness, and the bone mass of the mandible. Oral Surg Oral Med Oral Pathol Oral Radiol Endo 2001;92:346-52.

[7] White SC, Rudolph DJ. Alterations of the trabecular pattern of the jaws in patients with osteoporosis. Oral Surg Oral Med Oral Pathol Oral Radiol Endo 1999;88:628-35.

[8] Shrout MK, Hildebolt CF, Potter BJ, Brunsden TKB, Pilgram TK, Dotson $\mathrm{M}$, et al. Comparison of morphological measurements extracted from digitized dental radiographs with lumbar and femoral bone mineral density measurements in postmenopausal women. J Periodontol 2000;71:335-40.

[9] Southard TE, Southard KA, Lee A. Alveolar process fractal dimension and postcranial bone density. Oral Surg Oral Med Oral Pathol Oral Radiol Endo 2001;91:486-91.

[10] Klemetti E, Kolmakov S, Kröger H. Pantomography in assessment of the osteoporosis risk group. Scand J Dent Res 1994;102:68-72.

[11] Taguchi A, Suei Y, Ohtsuka M, Otani K, Tanimoto K, Ohtaki M. Usefulness of panoramic radiography in the diagnosis of postmenopausal osteoporosis in women. Width and morphology of inferior cortex of the mandible. Dentomaxillofacial Radiol 1996;25:263-7.

[12] Tanaka M, Toyooka E, Kohno S, Ozawa H, Ejiri S. Long-term changes in trabecular structure of aged rat alveolar bone after ovariectomy. Oral Surg Oral Med Oral Pathol Oral Radiol Endo 2003;95:495-502.

[13] Hansen MA, Overgaard K, Riis BJ, Christiansen C. Role of peak bone mass and bone loss in postmenopausal osteoporosis: 12 year study. BMJ 1991;303:961-4.

[14] Von Wowern N. Bone mass of mandibles. In vitro and in vivo analyses. Dan Med Bull 1986;33:23-44.

[15] Jonasson G, Kiliaridis S. The association between the masseter muscle, the mandibular alveolar bone mass and thickness in dentate women. Arch Oral Biol 2004;49:1001-6.

[16] Lindh C, Petersson A, Rohlin M. Assessment of the trabecular pattern before endosseous implant treatment: diagnostic outcome of periapical radiography in the mandible. Oral Surg Oral Med Oral Pathol Oral Radiol Endo 1996;82:335-43.

[17] Jonasson G, Kiliaridis S, Gunnarsson R. Cervical thickness of the mandibular alveolar process and skeletal bone mineral density. Acta Odontol Scand 1999;57:155-61. 
[18] Houston WJB. The analysis of errors in orthodontic measurements. Am J Orthod 1983;83:382-90.

[19] Eriksen EF, Langdahl B, Vesterby A, Rungby J, Kassem M. Hormone replacement therapy prevents osteoclastic hyperactivity: a histomorphometric study in early postmenopausal women. J Bone Miner Res 1999;14:1217-21.

[20] Riggs BL, Khosla S, Atkinson EJ, Dunstan CR, Melton III LJ. Evidence that type I osteoporosis results from enhanced responsiveness of bone to estrogen deficiency. Osteoporos Int 2003;14:728-33.

[21] Kanis JA, Johnell O, Oden A, De Laet C, Mellstrom D. Diagnosis of osteoporosis and fracture threshold in men. Calcif Tissue Int 2001;69: 218-221.

[22] Von Wowern N. Variation in structure within the trabecular bone of the mandible. Scand J Dent Res 1977;85:613-22.

[23] Nilas L, Nörgård H, Pödenphant J, Gotfredsen A, Christiansen C. Bone composition in the distal forearm. Scand J Clin Lab Invest 1987;47:41-6.

[24] Warming L, Hassager C, Christiansen C. Changes in bone mineral density with age in men and women: a longitudinal study. Osteoporos Int 2002;13:105-12.

[25] Uusi-Rasi K, Sievänen H, Pasanen M, Oja P, Vuori I. Changes in bone mineral density during a mean 4-year follow up. In: Burckardt P, DawsonHughes B, Heaney PP, editors. Nutritional aspects of osteoporosis. San Diego: Academic Express; 2001. p. 65-73. Chapt. 7.

[26] Weinstein RS, Hutson MS. Decreased trabecular width and increased trabecular spacing contribute to bone loss with aging. Bone 1987; 8:137-42.

[27] Van der Stelt PF. Experimentally produced bone lesions. Oral Surg Oral Med Oral Pathol 1985;59:306-12.

[28] Couture RA, Whiting BR, Hildebolt CF, Dixon DA. Visibility of trabecular structures in oral radiographs. Oral Surg Oral Med Oral Pathol Oral Radiol Endo 2003;96:764-71.

[29] Parsell DE, Gatewood RS, Watts JD, Streckfus CF. Sensitivity of various radiographic methods for detection of oral cancellous bone lesions. Oral Surg Oral Med Oral Pathol Oral Radiol Endo 1998;86:498-502.

[30] Wilmore JH. The aging of bone and muscle. Clin Sports Med 1991;10:231-44.

[31] Mavropoulos A, Kiliaridis S, Bresin A, Ammann P. Effect of different masticatory functional and mechanical demands on the structural adaptation of the mandibular alveolar bone in young growing rats. Bone 2004;35:191-7.

[32] Von Wowern N, Hjorting-Hansen E, Stoltze K. Changes in bone mass in rat mandibles after tooth extraction. Int J Oral Surg 1979;87:229-33.

[33] Elovic RP, Hipp JA, Hayes WC. Maxillary molar extraction causes increased bone loss in the mandible of ovariectomized rats. J Bone Miner Res 1995;10:1087-93.

[34] Jonasson G, Kiliaridis S. Changes in the bucco-lingual thickness of the mandibular alveolar process and skeletal bone mineral density in dentate women: a five-year prospective study. Eur J Oral Sci 2005;113:114-20.

[35] Araujo MG, Lindhe J. Dimensional ridge alterations following tooth extraction. An experimental study in the dog. J Clin Periodontol 2005;32:212-8.

[36] Botticelli D, Berglundh T, Lindhe J. Hard-tissue alterations following immediate implant placement in extraction sites. J Clin Periodontol 2004;31:820-8.

[37] Enlow DH, Bianco HJ, Eklund S. The remodeling of the edentulous mandible. J Prosthet Dent 1976;36:685-93.

[38] Ammann P, Rizzoli R. Bone strength and its determinants. Osteoporos Int 2003;14(Suppl 3):S13-8.

[39] Kingsmill VJ, Boyde A. Variation in the apparent density of human mandibular bone with age and dental status. J Anat 1998;192:233-44.

[40] White SC. Oral radiographic predictors of osteoporosis. Dentomaxillofacial Radiol 2002;31:84-92.

[41] Friberg B, Jemt T, Lekholm U. Early failures in 4,641 consecutively placed Brånemark dental implants: a study from stage 1 surgery to the connection of completed prostheses. Int J Oral Maxillofac Implants 1991;6:142-6.

[42] Friberg B, Ekestubbe A, Mellstrom D, Sennerby L. Branemark implants and osteoporosis: a clinical exploratory study. Clin Implant Dent Relat Res 2001;3:50-6.

[43] Goldie RS, King GJ. Root resorption and tooth movement in orthodontically treated, calcium-deficient, and lactating rats. Am J Orthod 1984;85:424-30.

[44] Bridges T, King G, Mohammad A. The effect of age on tooth movement and mineral density in the alveolar tissue of the rat. Am J Orthod Dentofac Orthop 1988;93:245-50. 